\title{
Early Changes in Rat Diaphragm Biology with Mechanical Ventilation
}

\author{
Gábor Z. Rácz, Ghislaine Gayan-Ramirez, Dries Testelmans, Pascal Cadot, Kristel De Paepe, Ernő Zádor, \\ Frank Wuytack, and Marc Decramer \\ Respiratory Muscle Research Unit, Laboratory of Pneumology and Respiratory Division; Laboratory of Physiology; Laboratory for \\ Experimental Immunology, Katholieke Universiteit Leuven, Leuven, Belgium; and Institute of Biochemistry, Faculty of Medicine, \\ University of Szeged, Szeged, Hungary
}

\begin{abstract}
To better characterize the effects of 24-hour mechanical ventilation on diaphragm, the expression of myogenic transcription factors, myosin heavy chains, and sarcoplasmic/endoplasmic reticulum calcium-ATPase pumps was examined in rats. In the diaphragm of mechanically ventilated animals, the mRNA of MyoD, myosin heavy chain-2a and $-2 b$, and sarcoplasmic/endoplasmic reticulum calciumATPase-1a decreased, whereas myogenin mRNA increased. In the diaphragm of anesthetized and spontaneously breathing rats, only the mRNA of MyoD and myosin heavy chain-2a decreased. MyoD and myogenin protein expression followed the changes at the mRNA, whereas the myosin heavy chain isoforms did not change. Parallel experiments involving the gastrocnemius were performed to assess the relative contribution of muscle shortening versus immobilization-induced deconditioning on muscle regulatory factor expression. Passive shortening produced no additional effects compared with immobilization-induced deconditioning. The overall changes followed a remarkably similar pattern except for MyoD protein expression, which increased in the gastrocnemius and decreased in the diaphragm while its mRNA diminished in both muscles. The early alterations in the expression of muscle protein and regulatory factors may serve as underlying molecular basis for the impaired diaphragm function seen after 24 hours of mechanical ventilation. Whether immobilization-induced deconditioning and/ or passive shortening play a role in these alterations could not be fully unraveled.
\end{abstract}

Keywords: mechanical ventilation; myogenic regulatory factors; myosin heavy chain; diaphragm; passive shortening

Mechanical ventilation is commonly used in the management of respiratory failure. In $20-50 \%$ of patients after successful treatment of the original illness, weaning from mechanical ventilation may be problematic (1). Respiratory muscle dysfunction is the major factor in producing weaning problems.

Recently, animal models of mechanical ventilation were developed in which the effects of anesthesia and the effects produced by mechanical ventilation were distinguished. At first, it was shown that already 12-24 hours of mechanical ventilation resulted in reduced forces generated by the diaphragm in vitro $(2,3)$. The magnitude of diaphragmatic dys-

(Received in original form June 10, 2002; accepted in final form April 15, 2003) Supported by "Fonds voor Wetenschappelijk Onderzoek-Vlaanderen" grants G.0175.99 and G.0237.01, KULeuven Research Foundation OT 98/27, BIL 99/19 Ministerie Vlaamse Gemeenschap (Belgium), B-3/99 from the Ministry of Culture and Education (Hungary), and Astra-Zeneca Pharmaceuticals. G.G-R. is a postdoctoral fellow of the Fonds voor Wetenschappelijk Onderzoek-Vlaanderen.

Correspondence and requests for reprints should be addressed to Marc Decramer, M.D., Ph.D., Respiratory Division, University Hospital, Herestraat 49, B-3000 Leuven, Belgium. E-mail: marc.decramer@uz.kuleuven.ac.be

This article has an online supplement, which is accessible from this issue's table of contents online at www.atsjournals.org

Am J Respir Crit Care Med Vol 168. pp 297-304, 2003

Originally Published in Press as DOI: 10.1164/rccm.200206-5410C on April 17, 2003

Internet address: www.atsjournals.org function increased with the time spent on the ventilator $(3,4)$. Conversely, maximal transdiaphragmatic pressure generated during phrenic nerve stimulation was decreased after 24 hours of controlled mechanical ventilation and myofibril damage occurred after 3 days (4). Furthermore, a decrease in diaphragm fiber cross-section was noticed as early as after 18-24 hours of controlled mechanical ventilation, although the diaphragm mass remained unchanged $(2,5)$. The concomitantly decreased diaphragm protein levels, increased proteasome activity (5), and reduced insulin-like growth factor-I mRNA levels (2) suggest that an atrophy process would likely follow.

The question how mechanical ventilation affects the inspiratory muscles in patients with normal or already curtailed inspiratory muscle function appears to be of great clinical relevance but is difficult to assess in patients. Therefore, this study was performed (1) to further characterize the effects of mechanical ventilation on the diaphragm in terms of expression of transcription factors and key muscle proteins and (2) to unravel which of the consequences of mechanical ventilation, immobilization-induced deconditioning, or rhythmic passive shortening might be responsible for these effects. To address the first question, we measured the mRNA levels and/ or the protein levels of several factors that possibly influence diaphragm function directly or indirectly. These factors included (1) myogenic regulatory factors, as these transcription factors are known to stimulate myogenesis (6) and the change in $\mathrm{MyoD} /$ myogenin ratio may drive muscle fiber phenotype adaptation (7-9); (2) the Id-type inhibitors of DNA-binding protein that are known inhibitors of myogenesis (10) (indeed, the inhibitor of DNA-binding protein-1 has been proposed as a mediator of muscle disease atrophy [11]); (3) myosin heavy chain and sarcoplasmic/endoplasmic reticulum calcium ATPase isoforms, as they are two of the main proteins having an impact on, respectively, the rate of muscle force development and relaxation; and (4) acetylcholine receptor, as it is a good marker of decreased nerve-evoked electrical activity (12). In particular, the expression of the acetylcholine-receptor $\alpha$-subunit was found to be regulated by myogenic factors (13-15).

To address the second question, the gastrocnemius muscle was either immobilized or subjected to passive shortening in the same range as experienced by the diaphragm during mechanical ventilation.

We hypothesized that (1) mechanical ventilation would alter the expression levels of contractile proteins and transcription factors and (2) immobilization-induced deconditioning and/or passive shortening would be implicated in the diaphragm alterations seen after mechanical ventilation.

\section{METHODS}

\section{Experimental Procedures and Study Design}

Two series of experiments were conducted. First, we examined the consequences of 24 hours of mechanical ventilation on the expression of several factors in the diaphragm. Second, we determined the effects of 24 hours of 
rhythmic passive shortening and immobilization-induced deconditioning on the expression levels of these factors in the gastrocnemius. The mRNA and/or the protein expression levels of a number of factors were examined in the diaphragm and the gastrocnemius. These factors include the muscle regulatory factors (myogenic determination or MyoD, myogenin, myf-5; herculin or MRF4), the inhibitors of DNA-binding proteins, the myosin heavy chain isoforms, the sarcoplasmic/endoplasmic reticulum calcium ATPase pumps, and acetylcholine receptor $\alpha$-subunit.

Effects of mechanical ventilation on the diaphragm. In the mechanical ventilation study, 52 male Wistar rats were randomly divided into three groups: (1) a control group, where no intervention was performed $(\mathrm{n}=10) ;(2)$ anesthetized and spontaneously breathing rats in which the same surgery was performed as in the animals under mechanical ventilation $(\mathrm{n}=26)$; and $(3)$ anesthetized and mechanically ventilated animals $(\mathrm{n}=16)$. These were the same animals as in a previous publication (2).

Effects of immobilization and rhythmic passive shortening on the gastrocnemius. In this study, 12 animals were instrumented as in the mechanical ventilation study, but in addition, both hindlimbs were immobilized. Whereas the right hindlimb was only immobilized, the left hindlimb was also passively moved rhythmically (Figure 1). A ventilator (Harvard pump) was adapted such that it allowed attachment to the left hindlimb of the rat. Movements of the piston were translated into movements of the foot, producing thereby passive shortening of the gastrocnemius at 55 movements per minute. The device was calibrated such that the degree of shortening was approximately $10 \%$ of resting muscle length, a change in length similar to the one undergone by the diaphragm during mechanical ventilation (16). The moved hindlimb was compared with the contralateral side, which was immobilized at resting position and underwent the effects of anesthesia and immobilization-induced deconditioning. The gastrocnemius muscle from the freely moving and ad libitum fed animals from the mechanical ventilation study served as the true control group.

Common procedures. Except for the control rats, all animals were initially anesthetized with sodium pentobarbital and were tracheotomized, and their body temperature was continuously monitored with an internal probe and maintained at $37^{\circ} \mathrm{C}$. During the 24 hours, continuous infusion of anestheticum and heparin was given via the right jugular vein and carotid artery, respectively, using pressure pumps (Pilot A2; Fresenius, Schelle, Belgium). Constant levels of anesthesia were controlled throughout the experiment by evaluating foot reflex, corneal reflex, arterial blood pressure, and breathing pattern (for the spontaneously breathing group). Animals also received enteral nutrition, including vitamins and minerals that were administered via a gastric tube. Animals breathed humidified air enriched with $\mathrm{O}_{2}$ and maintained at $37^{\circ} \mathrm{C}$. Me-

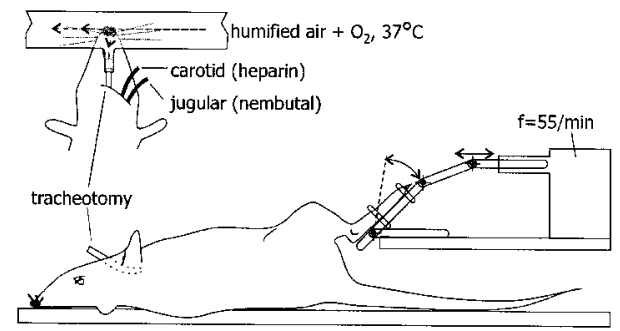

Figure 1. Experimental setup of the rhythmic passive shortening study. The rat is tracheotomized and breathed humidified air enriched with $\mathrm{O}_{2}$ and maintained at $37^{\circ} \mathrm{C}$. During the 24 hours, continuous infusion of anestheticum (nembutal) and heparin was given via the right jugular vein and carotid artery, respectively. In addition, both hindlimbs were immobilized, but while the right hindlimb was solely immobilized (data not shown), the left hindlimb was also passively moved rhythmically. Passive shortening of the left hindlimb occurred at 55 movements per minute ( $f=$ frequency). The device was calibrated such that the degree of gastrocnemius shortening was approximately $10 \%$ of resting muscle length, a change in length similar to the one experienced by the diaphragm during mechanical ventilation. chanically ventilated rats were ventilated with a respiratory rate of 55 breaths per minute.

After completion of the 24 hours, blood gas analysis was performed while diaphragm and gastrocnemius samples were removed and frozen in liquid nitrogen.

\section{Histologic Procedure: Mechanical Ventilation Study Only}

For the mechanical ventilation study, a diaphragm sample was fixed to a cork holder with its fibers oriented perpendicularly to the surface and was frozen in isopentane cooled in liquid nitrogen. Serial cross-sections parallel to the cork were stained with hematoxylin and eosin. Qualitative examination of these slides was performed by an expert who was not aware of the experimental design of the study.

\section{mRNA Extraction and Reverse Transcriptase-Polymerase Chain Reaction}

Total RNA of diaphragm and gastrocnemius was isolated using the guanidinium isothiocyanate procedure with cesium chloride method (17), as previously described (18). After reverse transcriptase reaction (Gibco BRL ThermoScript kit; Life Technologies, Merelbeke, Belgium), the first-strand cDNA mixture was subjected to polymerase chain reaction (Perkin Elmer kit, Lennik, Belgium). The number of polymerase chain reaction cycles was adjusted to avoid saturation of the amplification system. Amplification products were identified by their sizes after electrophoresis on $6 \%$ (wt/vol) acrylamide gels. After staining with Vistra Green, the fluorescence levels of the bands were quantified by means of a PhosphorImager model 425 (Molecular Dynamics, Sunnyvale, CA). Band intensities of the amplified fragments were normalized to the corresponding cyclophilin A (diaphragm) or L32 (gastrocnemius) amplification signals. No attempts were made to quantify the amount of the different target mRNAs, as reverse transcriptase-polymerase chain reaction is only a semiquantitative technique; only relative measurements are reported in the text.

\section{Myosin Heavy Chain Extraction and Electrophoresis}

Myosin heavy chain isoforms from the diaphragm and gastrocnemius from both studies were extracted as previously described (19). Sodium dodecyl sulfate-polyacrylamide gel electrophoresis with an $8 \%$ separating gel containing $30 \%$ glycerol and a $4 \%$ stacking gel was performed during 24 hours at $270 \mathrm{~V}$ and $4^{\circ} \mathrm{C}$. The gels were stained with silver nitrate (BioRad Silver stain plus kit; BioRad, Richmond, CA), air dried, and scanned. Bands were quantified using a gel documentation software system (Quanti One software; BioRad).

\section{Western Blot Quantification of MyoD and Myogenin}

Frozen muscle samples were homogenized according to the protocol of Sakuma and colleagues (20). Proteins ( $20 \mathrm{or} 50 \mu \mathrm{g})$ were separated by sodium dodecyl sulfate-polyacrylamide gel electrophoresis $(4 \%$ stacking gel and $12.5 \%$ separating gel) and then transferred onto polyvinyldifluoride membranes by semidry blotting. The blots were blocked and incubated with polyclonal rabbit anti-MyoD (sc-760; Santa Cruz Biotechnology, Santa Cruz, CA) and antimyogenin (sc-576; Santa Cruz Biotechnology). Horseradish peroxidase-conjugated rat anti-rabbit IgG (Biosource, Nivelles, Belgium) was used as secondary antibody. After revelation of the bands with tetramethylbenzidine, the blots were scanned and quantified using the Quanti One software (BioRad).

\section{Statistical Analysis}

The individual reverse transcriptase-polymerase chain reaction results were normalized to the housekeeping gene mRNA data. All data are presented as mean \pm SE. Differences between groups were assessed with one-way analysis of variance followed by Newman-Keuls post hoc test using the GraphPad Prism 3 software.

\section{RESULTS}

\section{General Findings}

Mortality. In the mechanical ventilation study, 12 animals out of the 16 studied survived the 24 hours in the mechanically ventilated 
group, whereas only 9 out the 26 studied survived in the spontaneously breathing group. Thus, although mortality was $25 \%$ in the mechanically ventilated rats, it was higher in the spontaneously breathing group (65\%) because in the latter group overdosing of anesthesia led to apnea and death. For the rhythmic passive shortening study, 10 out of 12 animals survived such that the mortality rate was $16 \%$.

Blood gases and arterial blood pressure. Blood gas analysis showed that $\mathrm{PA}_{\mathrm{O}_{2}}$ remained in the normal range for the anesthetized animals in the mechanical ventilation study (spontaneously breathing rats, $123 \pm 13$; and mechanically ventilated rats, $142 \pm$ $13 \mathrm{~mm} \mathrm{Hg}$ ) and in the rhythmic passive shortening study (113 \pm $6 \mathrm{~mm} \mathrm{Hg}$ ). This was also the case for $\mathrm{PA}_{\mathrm{CO}_{2}}$ (spontaneously breathing rats, $31 \pm 5$; mechanically ventilated rats, $33 \pm 5$; and rhythmic passive shortening study, $42 \pm 2 \mathrm{~mm} \mathrm{Hg}$ ) and $\mathrm{pH}$ (spontaneously breathing rats, $7.38 \pm 0.02$; mechanically ventilated rats, $7.45 \pm 0.04$; and rhythmic passive shortening study, $7.37 \pm 0.02)$.

Arterial blood pressure was similar in the different groups and averaged $130 \pm 7,104 \pm 11$, and $128 \pm 8 \mathrm{~mm} \mathrm{Hg}$ for the spontaneously breathing, mechanically ventilated groups, and the rhythmic passive shortening study, respectively.

\section{Histological Analysis: Mechanical Ventilation Study Only}

Qualitative examination of the diaphragm sections stained with hematoxylin and eosin did not reveal any abnormalities in diaphragm histology whatever the condition except for the fact that diaphragm fiber dimensions were smaller in the spontaneously breathing and mechanically ventilated groups.

\section{Reverse Transcriptase-Polymerase Chain Reaction Data}

\section{Myogenic regulatory factors.}

Mechanical ventilation study. Compared with control group, diaphragm myogenin mRNA levels were significantly increased in the mechanically ventilated group $(67 \%, \mathrm{p}<0.01$ vs. control group), whereas they tended to increase in the spontaneously breathing group (32\%, $\mathrm{p}=\mathrm{NS}$ ) (Figure 2A). Myf-5 mRNA increased together with myogenin after mechanical ventilation
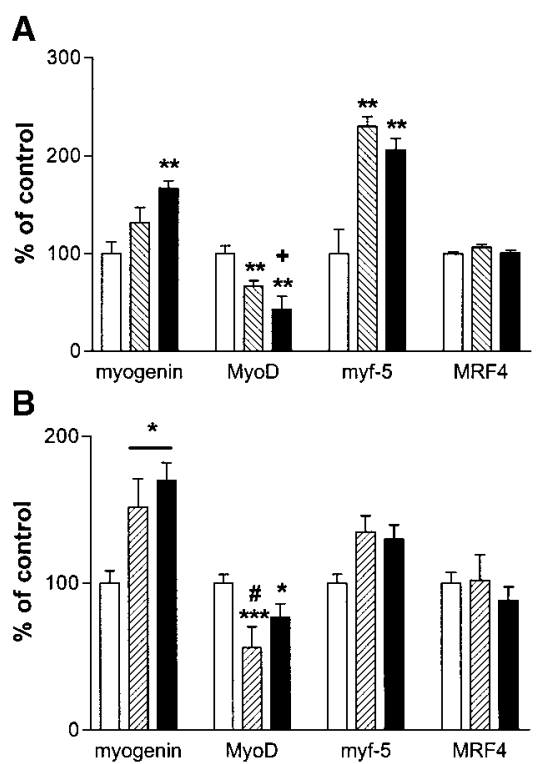

Figure 2. mRNA levels of myogenic regulatory factors determined by reverse transcriptase-polymerase chain reaction in the diaphragm $(A)$ of control rats (open bars, $\mathrm{n}=$ 9), spontaneously breathing rats (hatched bars, $\mathrm{n}=9$ ), and rats under mechanical ventilation (solid bars, $\mathrm{n}=12$ ) and in the gastrocnemius $(B)$ of control rats (open bars, $\mathrm{n}=9)$, after immobilization (hatched bars, $\mathrm{n}=$ 10), and after both immobilization and passive shortening (solid bars, $\mathrm{n}=10$ ). Values were normalized to the corresponding house-keeping gene amplification signals. Values are means and SE. ${ }^{*} p<0.05$, ${ }^{* *} p<0.01$, and ${ }^{* * *} p<$ 0.001 versus control; ${ }^{+} p<0.05$ versus spontaneously breathing; ${ }^{\#} p<$ 0.05 versus immobilization and passive shortening.
(107\%, p < 0.01 vs. control group); however, it also increased significantly in the spontaneously breathing group $(130 \%, \mathrm{p}<$ 0.01 vs. control group) (Figure 2A). In contrast, the level of MyoD mRNA decreased both in the spontaneously breathing rats $(-33 \%, \mathrm{p}<0.01$ vs. control group) and even more so in mechanically ventilated animals $(-56 \%, \mathrm{p}<0.001$ vs. control group), the latter being also statistically significantly different from the spontaneously breathing group $(\mathrm{p}<0.05)$ (Figure $2 \mathrm{~A})$. As a consequence, compared with control group, the MyoD/ myogenin ratio, which may reflect the ongoing changes in isoform switch, decreased significantly in the spontaneously breathing group $(-34 \%, \mathrm{p}<0.05$ vs. control group) and more particularly after mechanical ventilation $(-70 \%, \mathrm{p}<0.001$ vs. control group) (Figure 3A). In addition, this ratio was significantly decreased by $53 \%$ in the mechanically ventilated group compared with the spontaneously breathing group ( $p<0.01$ vs. spontaneously breathing). mRNA levels of MRF4, a factor involved in the later stages of myogenic transformation pathway, remained unchanged (Figure 2A).

Rhythmic passive shortening study. Passive shortening combined with immobilization caused a significant reduction in gastrocnemius MyoD mRNA only $(-23 \%, p<0.05$ vs. control group). However, after immobilization alone, a slightly more pronounced decay was observed $(-44 \%$, p $<0.001$ vs. control group, and $-21 \%, \mathrm{p}<0.05$ vs. immobilization and passive shortening) (Figure 2B). It is worth to note that myogenin (immobilization, 52\%; immobilization and passive shortening, $70 \%$, p < 0.05 vs. control group) and myf-5 (immobilization, $34 \%$; immobilization and passive shortening, 29\%; $\mathrm{p}=$ NS) mRNA increased after immobilization and also after passive shortening (Figure $2 \mathrm{~B})$, as was also the case in the diaphragm of mechanically ventilated and spontaneously breathing groups (Figure 2A). As a result, the MyoD/myogenin ratio decreased similarly after immobilization $(-60 \%, \mathrm{p}<0.001$ vs. control group) and after immobilization combined with passive shortening $(-51 \%, \mathrm{p}<$ 0.001 vs. control group) (Figure 3B). MRF4 mRNA did not change whatever the condition.

Inhibitor of the DNA-binding protein isoforms. Mechanical ventilation study. Reverse transcriptase-polymerase chain reaction data showed that inhibitor of DNA-binding protein-1 mRNA levels were significantly and equally reduced both in the diaphragm of mechanically ventilated and spontaneously breathing animals $(-30 \%, \mathrm{p}<0.001)$, whereas inhibitor of DNA-binding protein-2 and inhibitor of DNA-binding protein-3 mRNA showed no changes, and inhibitor of DNA-binding protein-4 mRNA was not detectable.

Rhythmic passive shortening study. In the immobilized and passively moved but also in the solely immobilized gastrocnemius, inhibitor of DNA-binding protein-3 mRNA decreased equally ( $-27 \%$ and $-25 \%$, respectively, $\mathrm{p}<0.01$ vs. control group), whereas the other inhibitor of DNA-binding protein isoform mRNA did not change.

Myosin heavy chain isoforms: mechanical ventilation study only. The fast myosin heavy chain-2a mRNA decreased in the diaphragm of the spontaneously breathing group $(-10 \%, \mathrm{p}<0.05$ vs. control group) and even more so in the mechanically ventilated group $(-20 \%, \mathrm{p}<0.001$ vs. control group). The decrease in the latter was moreover significantly different from the spontaneously breathing group $(\mathrm{p}<0.05)$ (Figure $4 \mathrm{~A})$. Also myosin heavy chain-2b mRNA decreased, but this reached statistical significance only in the mechanically ventilated animals $(-19 \%$, $\mathrm{p}<0.05$ vs. control group) (Figure 4A). Myosin heavy chain-1 and myosin heavy chain-2x mRNA did not change during the experiment (Figure 4A).

Sarcoplasmic/endoplasmic reticulum calcium ATPase isoforms: mechanical ventilation study only. Sarcoplasmic/endoplasmic re- 
A
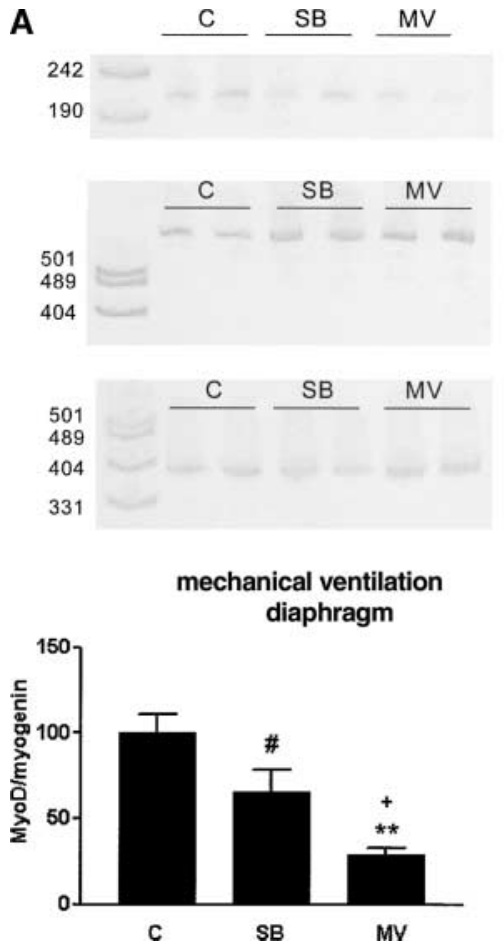

B
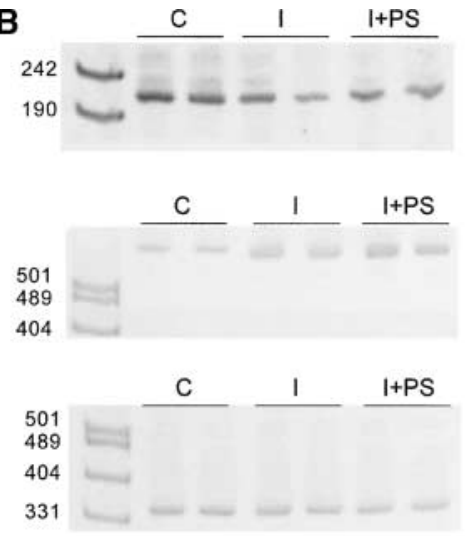

passive gastrocnemius shortening

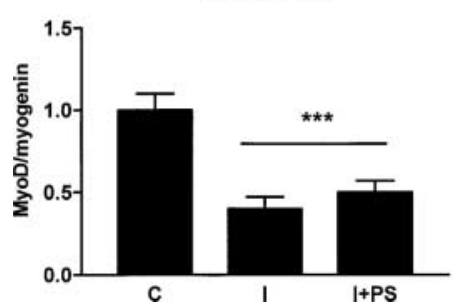

Figure 3. MyoD/myogenin ratio in the diaphragm $(A)$ of control rats $(C, n=9)$, spontaneously breathing rats $(S B, n=9)$, and rats under mechanical ventilation (MV, $\mathrm{n}=12)$ and in the gastrocnemius $(B)$ of control rats $(C, n=9)$, after immobilization $(I, n=12)$, and after both immobilization and passive shortening $(I+P S, n=12)$. Representative reverse transcriptase-polymerase chain reaction results are provided for MyoD, myogenin, and housekeeping gene (cyclophilin A for the diaphragm and L32 for the gastrocnemius). Each lane represents a sample with the first lane being the molecular marker. The values of the bar graph were normalized to the corresponding housekeeping gene amplification signals. Values are means and SE. ${ }^{*} p<0.05,{ }^{* * *} p<0.001$ versus $\mathrm{C},{ }^{+} \mathrm{p}<0.05$ versus $\mathrm{SB}$.

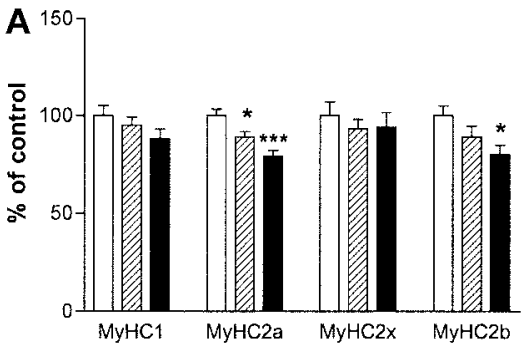

Figure 4. mRNA levels of myosin heavy chain isoforms (MyHC) (A), sarcoplasmic/endoplasmic reticulum calcium ATPase (SERCA) isoforms, and acetylcholine receptor $\alpha$-subunit $(\mathrm{AChR}-\alpha)$ (B) determined by reverse transcriptase-polymerase chain reaction in the diaphragm of contro rats (open bars, $\mathrm{n}=9$ ), spontaneously breathing rats (hatched bars, $\mathrm{n}=9$ ), and rats under mechanical ventilation (solid bars, $\mathrm{n}=12$ ). Values were normalized to the corresponding housekeeping gene amplification signals. Values are means and SE. ${ }^{*} p<0.05$ and ${ }^{* * *} p<0.001$ versus controls.

ticulum calcium ATPase-1a mRNA, which is characteristic of the fast muscle fibers, decreased significantly only in the diaphragm of the mechanically ventilated group $(-21 \%, p<0.05$ vs. control group) (Figure 4B). The slow-type sarcoplasmic/endoplasmic reticulum calcium ATPase-2a mRNA did not change in the experiment (Figure 4B).

Acetylcholine receptor $\alpha$-subunit: mechanical ventilation study only. The mRNA level of acetylcholine receptor $\alpha$-subunit showed a marked increase in the diaphragm of the spontaneously breathing $(140 \%)$ and mechanically ventilated $(120 \%)$ groups (Figure 4B). These increases reached statistical significance with one-way analysis of variance $(p<0.05)$ but not with the Newman-Keuls post hoc test.

\section{Myosin Heavy Chain Electrophoresis}

For both studies, there were no changes in the relative proportion of the different myosin heavy chain isoforms between the different groups. For each study, the proportions of the different myosin heavy chain isoforms are reported in Table 1.

\section{Western Blot: Analysis of MyoD and Myogenin}

Mechanical ventilation study. Compared with the control group, diaphragm MyoD was significantly reduced in mechanically ventilated group $(-49 \%, p<0.05$ vs. control group and spontaneously breathing) and remained unchanged in spontaneously breathing animals (Figure 5), whereas myogenin tended to increase (spontaneously breathing, 115\%; mechanically ventilated, 59\%; $\mathrm{p}=0.08)$.

Rhythmic passive shortening. Compared with the control group, gastrocnemius MyoD was significantly increased after immobilization ( $40 \%, \mathrm{p}<0.01$ vs. control group) and also after immobilization and passive shortening ( $52 \%, \mathrm{p}<0.001 \mathrm{vs.} \mathrm{control} \mathrm{group).}$ For myogenin, a tendency to increase was also observed in both groups (immobilization, 36\%; immobilization and passive shortening, 44\%) compared with the control group, but these increases failed to reach statistical significance. These changes in protein expression thus did not follow the changes in mRNA expression for MyoD, whereas for myogenin, a close accordance with the mRNA changes was present. 
TABLE 1. PERCENTAGE OF THE DIFFERENT MYOSIN HEAVY CHAIN ISOFORMS IN THE DIAPHRAGM OF THE MECHANICAL VENTILATION STUDY AND IN THE GASTROCNEMIUS OF THE PASSIVE SHORTENING STUDY

\begin{tabular}{|c|c|c|c|}
\hline & \multicolumn{3}{|c|}{ Mechanical Ventilation: Diaphragm } \\
\hline & Myosin Heavy Chain-1 & Myosin Heavy Chain-2a & Myosin Heavy Chain-2x \\
\hline Control group & $25 \pm 1$ & $41 \pm 2$ & $34 \pm 2$ \\
\hline Spontaneous breathing & $23 \pm 1$ & $39 \pm 1$ & $37 \pm 1$ \\
\hline \multirow[t]{3}{*}{ Mechanical ventilation } & $22 \pm 1$ & $41 \pm 2$ & $37 \pm 1$ \\
\hline & \multicolumn{3}{|c|}{ Passive Shortening: Gastrocnemius } \\
\hline & Myosin Heavy Chain-1 & Myosin Heavy Chain-2a & Myosin Heavy Chain-2x \\
\hline Control group & $33 \pm 3$ & $32 \pm 2$ & $35 \pm 7$ \\
\hline Immobilization & $38 \pm 2$ & $27 \pm 1$ & $35 \pm 1$ \\
\hline Immobilization and passive shortening & $34 \pm 1$ & $31 \pm 1$ & $35 \pm 1$ \\
\hline
\end{tabular}

Values are mean \pm SE. Data are expressed as percentages.

\section{DISCUSSION}

This study showed that anesthesia, but more particularly controlled mechanical ventilation, alters the mRNA levels of the muscle regulatory factors (MyoD, myogenin), the myosin heavy chain isoforms, and the sarcoplasmic/endoplasmic reticulum calcium ATPase isoforms as early as after 24 hours. The overall pattern of these changes points in the direction of a reduction in the mRNA levels of the fast isoforms of the respective muscle proteins (myosin heavy chain and sarcoplasmic/endoplasmic reticulum calcium ATPase) paralleled by a reduction in the levels of MyoD, whereas the mRNA of myogenin, a factor that is often
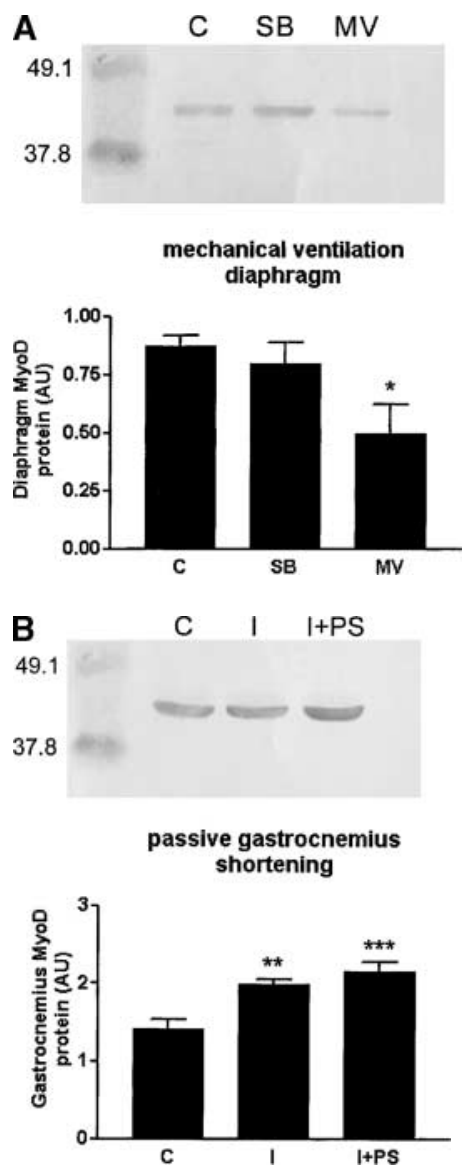

found together with the slow isoforms of the previously mentioned proteins, is increased. MyoD and myogenin protein expression followed the corresponding changes seen at the mRNA level, whereas no significant changes were noticed in the myosin heavy chain protein isoforms. In addition, our data on gastrocnemius showed that passive shortening did not further affect the expression levels of the studied factors compared with immobilizationinduced deconditioning. These mRNA and protein changes in gastrocnemius were similar in nature to those observed in the diaphragm after mechanical ventilation except for MyoD protein expression, which increased in the gastrocnemius but decreased in the diaphragm. Whether this increase in gastrocnemius MyoD protein is transient and will be followed by a decrease as expected from its mRNA expression is impossible to predict from these data.

The changes observed in this study are likely to be related to the effects of the mechanical ventilation, as blood gas levels and arterial blood pressure, which can influence muscle force and/or transcription rate, remained within the normal range. Infection was unlikely to develop over the 24-hour time period $(3,21)$. Although nutritional support was limited to $69 \mathrm{kcal} /$ day (corresponding to $173 \mathrm{kcal} / \mathrm{kg} /$ day) in the anesthetized animals, this caloric intake was similar to that used in the study of Powers and colleagues (3) and was much higher than in other animal models of mechanical ventilation where longer duration of mechanical ventilation was performed $(50 \mathrm{kcal} / \mathrm{kg} /$ day up to 4 days of mechanical ventilation [22], 30-35 kcal/kg/day up to 5 days of mechanical ventilation [23], and $100 \mathrm{cal} / \mathrm{kg} /$ day up to 1 or 3 days of mechanical ventilation [4]). That nutritional deprivation may have occurred in our model is possible, but the contribution of undernutrition is probably not essential, as it is well know that undernutrition does not reduce diaphragmatic maximal tetanic tension (24-27) nor does it affect diaphragmatic insulin-like growth factor-I mRNA (28), whereas both were observed in our model. Moreover, undernutrition even if present would not explain the differences between the spontaneously breathing rats and the rats under mechanical ventilation. Finally, the significant differences between spontaneous breathing and mechanically ventilated groups in the mRNA expression of MyoD/myogenin ratio, myosin heavy chain2a, myosin heavy chain-2b, sarcoplasmic/endoplasmic reticulum calcium ATPase-1a show that although anesthesia itself had an effect on diaphragm properties, it was markedly enhanced by mechanical ventilation.

The myogenic regulatory factors belong to the basic helixloop-helix family and are muscle specific. They form heterodimers with the E proteins, and because they contain a basic DNA- 
binding domain, they bind DNA in a sequence-specific manner at sites known as E boxes. Because this DNA motif is present in the promoters of many skeletal muscle-specific genes, gene activation is mediated in a myogenic regulatory factor-dependent manner. During embryogenesis, the myogenic regulatory factors stimulate myoblast determination and differentiation (29). The myogenic regulatory factors have been shown to be negatively regulated by the helix-loop-helix inhibitor of the DNA-binding protein, which lacks the basic DNA-binding domain (10). Although the role of the myogenic regulatory factors and the inhibitor of the DNA-binding proteins in adult mature skeletal muscles is not fully established, the expression levels of these transcription factors have been shown to be modulated by various types of intervention such as denervation, immobilization, electrostimulation, mechanical loading, and hormone treatment $(8,9,12,15)$.

\section{Mechanical Ventilation Study}

This study is the first showing that 24 hours of controlled mechanical ventilation leads to marked changes in the mRNA levels of key muscle proteins and regulatory factors. For the myosin heavy chain isoforms and the sarcoplasmic/endoplasmic reticulum calcium ATPase pumps, mechanical ventilation led to a reduction in the mRNA levels of the fast isoforms, these effects being independent of anesthesia. These alterations are expected to favor a slow oxidative profile. This is in line with a previous report showing that although all diaphragm fibers atrophied after 18 hours of controlled mechanical ventilation, the effect on type II fibers was greater than on type I fibers (5). Increases in citrate synthase activity in the diaphragm of mechanically ventilated rats further confirmed that oxidative capacity of the diaphragm increased after short-term controlled mechanical ventilation (30). In agreement with Shanely and colleagues (5), no significant changes in myosin heavy chain isoform distribution were observed in this study after 24 hours of controlled mechanical ventilation, whereas with more prolonged (3-7 days) mechanical ventilation combined with antibiotic treatment and neuromuscular blockade, an increased proportion of diaphragm fibers coexpressing myosin heavy chain-1 and -2 at the expense of the pure myosin heavy chain-1 population was reported in rats (22). Caution should, however, be taken, as antibiotics and neuromuscular blocking agents in addition to mechanical ventilation may have contributed to this phenotype adaptation, as they are known to affect inspiratory muscle contractility (31).

For the muscle regulatory factors, the alterations of MyoD and myogenin mRNA were clearly more pronounced after mechanical ventilation than during anesthesia. Although the role of the myogenic regulatory factors in adult muscle is not well defined, MyoD and myogenin have been reported to be implicated as regulators of fiber phenotype (32). In fact, MyoD mRNA is predominantly located in fast twitch muscle, whereas myogenin mRNA is mainly found in slow-twitch muscle (8). Although still controversial, several studies have shown that the MyoD/myogenin is highly correlated with muscle fiber phenotype such that a transformation from slow to fast phenotype was associated with a decreased myogenin and elevated MyoD mRNA expression (32). Thus, the reduction in the MyoD/myogenin ratio after mechanical ventilation both at the mRNA and the protein levels suggests phenotypic adaptation toward a slower profile. This is in line with the effects observed in this study for the myosin heavy chain isoforms and sarcoplasmic/endoplasmic reticulum calcium ATPase pumps at the mRNA levels. Especially the decrease in myosin heavy chain-2b mRNA observed after mechanical ventilation further underlines the already known strong correlation between MyoD and myosin heavy chain-2b gene expression (33). However, because of the low levels of myosin heavy chain-2b in the rat diaphragm, the functional implication of this decrease should be interpreted with care. No changes in myosin heavy chain isoforms were observed, however, at the protein level in the diaphragm of the mechanically ventilated rats despite alterations toward a slower profile at the mRNA levels. Alterations in MyoD and/or myogenin expression may induce adaptations in the intrinsic properties of the fiber without altering fiber phenotype $(34,35)$. That the diaphragm in mechanically ventilated rats moves toward a slower profile is further confirmed by the data of Shanely and colleagues showing increases in citrate synthase activity after 18 hours of controlled mechanical ventilation without changes in fiber proportion (5).

Furthermore, caution should also be taken because the role of $\mathrm{MyoD} / \mathrm{myogenin}$ in regulating myosin heavy chain isoform expression is model dependent, and whereas it seems to be valid for hindlimb unloading, it is not supported in tetrodotoxininduced paralysis or after short-term spinal cord transection (32). Moreover, although it would be expected that MyoD knockout mice would have reduced proportions of fast fibers, these mice showed a remarkably normal fiber-type distribution (36). Hughes and colleagues, however, showed that a loss of the MyoD function caused by gene disruption resulted in a subtle shift toward a slower profile in fast muscles (35). On the other hand, although mice overexpressing myogenin showed no alterations in myosin heavy chain proportion, a shift of enzyme activity from glycolytic to oxidative metabolism was reported in the muscles of these transgenic mice (34). Nonetheless, the diaphragm seems to behave differently than peripheral skeletal muscle, as recently MyoD deletion has been reported to result in a fast-to-slow shift in the myosin heavy chain phenotype of the diaphragm (37). This MyoD elimination was associated with a downward shift of the diaphragm force-frequency relationship and a decrease in the maximal tetanic tension. This further supports the view that the downregulation of MyoD seen in our study after mechanical ventilation may have contributed to the previously reported reduction in diaphragm function (2).

In this study, an increased myf-5 mRNA was also observed, but because this increase was similar in the spontaneous breathing animals and in the rats submitted to mechanical ventilation, it was likely to reflect the effect of anesthesia and/or surgical procedure. Intriguingly, we previously observed similar increases in myf-5 mRNA levels in the diaphragm after surgical procedure and anesthesia in a model of lung volume reduction surgery in hamsters (38). The functional implication of elevated myf-5 mRNA in these two models is, however, not known, but our present data show that this increase is not specific for mechanical ventilation effects. As MyoD mRNA decreased in our study in both anesthetized groups (but to a greater extent after mechanical ventilation), it might be suggested that the similar increase in myf-5 mRNA in the anesthetized animals represents a compensatory response of myf-5 for this MyoD downregulation. Indeed, in knockout MyoD mice, high levels of myf-5 mRNA have been reported to maintain normal muscle phenotype (36). This is further supported by the fact that the proportion of the myosin heavy chain isoforms was unaltered in this study. Finally, recent data suggest that like MyoD, myf-5 may also influence myosin heavy chain isoform expression (39). This further supports the idea that myf-5 may compensate for MyoD downregulation to maintain the normal diaphragm phenotype.

The decrease in the inhibitor of DNA-binding protein-1 mRNA observed in the present study was similar in spontaneously breathing and mechanically ventilated animals, suggesting thereby that this decrease was not caused by mechanical ventilation but was rather related to anesthesia and/or surgical procedure. Similar decreases in the inhibitor of DNA-binding protein-1 mRNA were previously observed in the hamster diaphragm after anesthesia and surgical procedure (38). As the inhibitors of DNA-binding protein are known to negatively regulate muscle growth, such a 
decrease in the inhibitor of DNA-binding protein-1 mRNA levels is expected to result in muscle hypertrophy. Whether this decrease in the inhibitor of DNA-binding protein-1 mRNA would be a compensatory mechanism in response to anesthesia and/or surgical procedure cannot be concluded from these data and is beyond the aim of this study.

\section{Rhythmic Passive Shortening Study}

This study is the first attempting to develop an animal model examine which of the consequences of mechanical ventilation (immobilization-induced deconditioning or rhythmic passive shortening) may be involved in the diaphragm dysfunction caused by mechanical ventilation. Our data showed that similar results were observed after gastrocnemius immobilization alone or combined with rhythmic passive shortening. More specifically, while MyoD mRNA was decreased, myogenin mRNA was increased as was myf-5 mRNA, although the latter did not reach statistical significance. In contrast, MyoD protein expression did not parallel the changes seen at the mRNA levels as MyoD significantly increased after immobilization and also after immobilization combined with rhythmic passive shortening. That MyoD protein increased while its mRNA decreased may reflect a transient response of MyoD protein that will be followed by a decrease in a later stage as expected from its RNA expression. The potential mechanisms responsible for the discrepancy between MyoD protein and mRNA are not known and are difficult to unravel especially because the regulation of MyoD is still not fully understood. Indeed, it is well known that MyoD is able to autoregulate its own transcription positively and that the turnover of both MyoD protein and mRNA is high (40). However, the function of this positive feedback loop in vivo is not clear. In fact, discrepancy between MyoD mRNA and protein has previously been reported in limb muscle after denervation $(41,42)$ and regeneration (43). Unfortunately, in none of these studies was a rationale for such discrepancy provided. We thus hypothesized that the increased MyoD protein observed in the gastrocnemius in this study may reflect a compensatory mechanism to the decrease in its mRNA to stabilize MyoD protein. On the other hand, knowing that MyoD turnover is high, MyoD effects are probably better related to its protein level rather than to its mRNA level.

The data of this study show that the gastrocnemius is affected by 24 hours inactivity but that the inactivity-induced effects are less pronounced than those observed in the diaphragm probably because more time is needed for the gastrocnemius to be similarly affected by inactivity as compared with the diaphragm. The nature of these effects is, however, similar in both muscles, except for MyoD protein expression, which increases in the gastrocnemius while it decreases in the diaphragm after mechanical ventilation. Whether this would lead to fiber phenotype adaptation at a later stage remains to be determined. Our data also show that 24 hours of gastrocnemius passive shortening did not produce any effects in addition to those induced by immobilizationinduced deconditioning whose effects developed rapidly and predominated during the first 24 hours at least. However, an effect of passive shortening should not be completely excluded as more time might be necessary for the stretch effect to develop.

Finally, caution should be taken when extrapolating conclusions from experiments on the gastrocnemius to the diaphragm, as many anatomical and functional differences exist between these two muscles. However, among all the skeletal muscles, the gastrocnemius was probably the most suitable muscle to examine immobilization-induced deconditioning and passive shortening. Indeed, first it best fits to the diaphragm for what concerns its fiber composition (25) and mRNA content of myogenic regulatory factors, especially for MyoD and myogenin (44). Fiber composition was a key factor to assess, as immobilization-induced deconditioning is known to preferentially affect slow-type muscle and to a lesser extent fast-type muscle. Thus, in order not to bias a potential effect, a mixed muscle such as the gastrocnemius was preferentially chosen to mimic better what happens to the diaphragm. Second, gastrocnemius access makes it easy to control for resting position and to adjust shortening during passive shortening. Third, the muscle is large enough to obtain sufficient material for several measurements. Of course, the activity patterns of the gastrocnemius and the diaphragm are very dissimilar, as the diaphragm has a longer duty cycle and is activated throughout the whole life and is likely to be particularly susceptible to the effects of, for example, inactivity (45).

On the other hand, because fiber length is a major issue, care was taken to induce immobilization and/or passive shortening of the gastrocnemius at a resting length that is known to be close to its optimal length. Of course, spatial arrangement and recruitment profiles are different between the two muscles; however, obviously, these parameters could not be controlled, and as such, they contribute to the limitations of the model. It is also true that $10 \%$ shortening may be more (or less) harmful for the diaphragm than for the gastrocnemius. However, it was reasonable to apply the same relative level of shortening to the gastrocnemius as would be experienced by the diaphragm during mechanical ventilation, as actually no literature is available to describe the effect of various extents of shortening on the gastrocnemius function. In fact, there is no skeletal muscle that would perfectly match the properties of the diaphragm, and for this reason, the data of gastrocnemius experiments should be interpreted in the perspective of the limitations of the study.

\section{Conclusions}

The data of this study emphasize that short-term mechanical ventilation results in important and multiple alterations of muscle protein and transcription factors at both the mRNA and also the protein levels. Part of these changes is likely to contribute to the diaphragm dysfunction caused by mechanical ventilation. Whether the effects of immobilization-induced deconditioning/ unloading and rhythmic passive shortening would play an important role in the diaphragm alterations seen after mechanical ventilation could not be fully unraveled by this study. Further studies are needed to map the complexity of this phenomenon to improve prevention of the weaning problems and to develop strategies to minimize the effects of mechanical ventilation on diaphragm function.

Acknowledgment: The authors sincerely thank Mr. Luc Grammet for his patience and expert work in building the setup for gastrocnemius passive shortening. They also thank Mrs. Leen Luyts for her excellent technical assistance.

\section{References}

1. Esteban A, Frutos F, Tobin MJ, Alia I, Solsona JF, Valverdu I, Fernandez $\mathrm{R}$, de la Cal MA, Benito S, Tomas R, et al. A comparison of four methods of weaning patients from mechanical ventilation. $N$ Engl J Med 1995;332:345-350.

2. Gayan-Ramirez G, de Paepe K, Cadot P, Decramer M. Detrimental effects of mechanical ventilation on diaphragm function and IGF-I mRNA in rats. Intensive Care Med 2003;29:825-833.

3. Powers SK, Shaneley RA, Coombes JS, Koesterer TJ, McKenzie M, Van Gammeren D, Cicale M, Dodd SL. Mechanical ventilation results in progressive contractile dysfunction in the diaphragm. J Appl Physiol 2002; $92: 1851-1858$.

4. Sassoon CSH, Caiozzo VJ, Manka A, Sieck GC. Altered diaphragm contractile properties with controlled mechanical ventilation. J Appl Physiol 2002;92:2585-2595.

5. Shanely RA, Lennon SL, Yimlamai T, Dodd S, Zergeroglu AM, Sugiura T, Enns D, Belcastro A, Powers SK. Mechanical ventilation-induced diaphragmatic atrophy is associated with oxidative injury and increased proteolytic activity. Am J Respir Crit Care Med 2002;165:1369-1374. 
6. Ludolph DC, Konieczny SF. Transcription factor families: muscling in on the myogenic program. FASEB J 1995;9:1595-1604.

7. Voytik SL, Przyborski M, Badylak SF, Konieczny SF. Differential expression of muscle regulatory factor genes in normal and denervated adult rat hindlimb muscles. Dev Dyn 1993;198:214-224.

8. Hughes SM, Taylor JM, Tapscott SJ, Gurley CM, Carter WJ, Peterson CA. Selective accumulation of MyoD and myogenin mRNAs in fast and slow adult skeletal muscle is controlled by innervation and hormones. Development 1993;118:1137-1147.

9. Mozdziak PE, Greaser ML, Schultz E. Myogenin, MyoD, and myosin heavy chain isoform expression following hindlimb suspension. Aviat Space Environ Med 1999;70:511-516.

10. Benezra R, Davis RL, Lockshon D, Turner DL, Weintraub H. The protein Id: a negative regulator of helix-loop-helix DNA binding proteins. Cell 1990;61:49-59.

11. Gundersen K, Merlie JP. Id-1 as a possible transcriptional mediator of muscle disuse atrophy. Proc Natl Acad Sci USA 1994;91:3647-3651.

12. Zador E, Dux L, Wuytack F. Prolonged passive stretch of rat soleus muscle provokes an increase in the mRNA levels of the muscle regulatory factors distributed along the entire length of the fibers. $J$ Muscle Res Cell Motil 1999;20:395-402.

13. Prody CA, Merlie JP. A developmental and tissue-specific enhancer in the mouse skeletal muscle acetylcholine receptor alpha-subunit gene regulated by myogenic factors. J Biol Chem 1991;266:22588-22596.

14. Merlie JP, Mudd J, Cheng TC, Olson EN. Myogenin and acetylcholine receptor alpha gene promotors mediate trancriptional regulation in response to motor innervation. J Biol Chem 1994;28:2461-2467.

15. Carlsen H, Gundersen K. Helix-loop-helix transcription factors in electrically active and inactive skeletal muscles. Muscle Nerve 2000;23: 1374-1380.

16. Decramer M, De Troyer A, Kelly S, Macklem PT. Mechanical arrangement of costal and crural diaphragm. J Appl Physiol 1984;56:1484-1490.

17. Chirgwin JM, Przybyla AE, McDonald RJ, Rutter WJ. Isolation of biologically active ribonucleic acid from sources enriched in ribonuclease. Biochemistry 1979;18:5294-5299.

18. Gayan-Ramirez G, Vanzeir L, Wuytack F, Decramer M. Corticosteroids decrease mRNA levels of SERCA pumps, whereas they increase sarcolipin mRNA in the rat diaphragm. $J$ Physiol (Lond) 2000;524:387-397.

19. Maréchal G, Coulton GR, Beckers-Bleukx G. Mechanical power and myosin composition of soleus and extensor digitorum longus muscle of ky mice. Am J Physiol 1995;268:C513-C519.

20. Sakuma K, Watanabe K, Sano M, Uramoto I, Totsuka T. Postnatal profiles of myogenic regulatory factors and the receptors of TGF-b2, LIF and IGF-I in the gastrocnemius and rectus femoris muscles of dy mouse. Acta Neuropathol (Berl) 2000;99:169-176.

21. Le Bourdelles G, Viires N, Boczkowski J, Seta N, Pavlovic D, Aubier M. Effects of mechanical ventilation on diaphragmatic contractile properties in rats. Am J Respir Crit Care Med 1994;149:1539-1544.

22. Yang L, Luo J, Bourdon J, Lin MC, Gottfried SB, Petrof BJ. Controlled mechanical ventilation leads to remodelling in the rat diaphragm. Am J Respir Crit Care Med 2002;166:1135-1140.

23. Radell PJ, Remahl S, Nichols DG, Eriksson LI. Effects of prolonged mechanical ventilation and inactivity on piglet diaphragm function. Intensive Care Med 2002;28:358-364.

24. Kelsen SG, Ference M, Kapoor S. Effects of prolonged undernutrition on structure and function of the diaphragm. $J$ Appl Physiol 1985;58: 1354-1359.

25. Dekhuijzen PNR, Gayan-Ramirez G, Bisschop A, De Bock V, Dom R, Decramer M. Corticosteroid treatment and nutritional deprivation cause a different pattern of atrophy in rat diaphragm. J Appl Physiol 1995;78:629-637.

26. Nava S, Gayan-Ramirez G, Rollier H, Bisschop A, Dom R, De Bock V, Decramer M. Effects of acute steroid administration on ventilatory and peripheral muscles in rats. Am J Respir Crit Care Med 1996;153: 1888-1896.

27. Lewis MI, LoRusso TJ, Zhan WZ, Sieck GC. Interactive effects of denervation and malnutrition on diaphragm structure and function. $J$ Appl Physiol 1996;81:2165-2172.

28. Gayan-Ramirez G, Vanderhoydonc F, Verhoeven G, Decramer M. Acute treatment with corticosteroids decreases IGF-I and IGF-II expression in the rat diaphragm and gastrocnemius. Am J Respir Crit Care Med 1999;159:283-289.

29. Sabourin LA, Rudnicki MA. The molecular regulation of myogenesis. Clin Genet 2000;57:16-25.

30. Shanely RA, Coombes JS, Zergeroglu AM, Webb KA, Powers SK. Shortduration mechanical ventilation enhances diaphragmatic fatigue resistance but impairs force production. Chest 2003;123:195-201.

31. Leatherman JW, Fluegel WL, David WS, Davies SF, Iber C. Muscle weakness in mechanically ventilated patients with severe asthma Am J Respir Crit Care Med 1996;153:1686-1690.

32. Talmadge RJ. Myosin heavy chain isoform expression following reduced neuromuscular activity: potential regulatory mechanisms. Muscle Nerve 2000;23:661-679.

33. Wheeler MT, Snyder EC, Patterson MN, Swoap SJ. A E-box within the MHC IIB gene is bound by MyoD and is required for gene expression in fast muscle. Am J Physiol 1999;276:C1069-C1078.

34. Hughes SM, Chi M-MY, Lowry OH, Gundersen K. Myogenin induces a shift of enzyme activity from glycolytic to oxidative metabolism in transgenic mice. J Cell Biol 1999;145:633-642.

35. Hughes SM, Koishi K, Rudnicki MA, Maggs AM. MyoD protein is differentially accumulated in fast and slow skeletal muscle fibres and required for normal fibre type balance in rodents. Mech Dev 1997;61: 151-163.

36. Rudnicki MA, Braun T, Hinuma S, Jaenisch R. Inactivation of MyoD in mice leads to up-regulation of the myogenic HLH gene myf-5 and results in apparently normal muscle development. Cell 1992;71:383-390.

37. Staib JL, Swoap SJ, Powers SK. Diaphragm contractile dysfunction in MyoD gene-inactivated mice. Am J Physiol Regul Integr Comp Physiol 2002;283:R583-R590.

38. Reynders V, Marchand E, Gayan-Ramirez G, De Leyn P, Verhoeven $\mathrm{G}$, Decramer M. Alterations in gene expression in hamster diaphragm after emphysema and lung volume reduction surgery. Eur Respir J 2002;19:1-8.

39. Muroya S, Nakajima I, Chikuni K. Related expression of MyoD and myf5 with myosin heavy chain isoform types in bovine adult skeletal muscles. Zoolog Sci 2002;19:755-761.

40. Thayer MJ, Tapscott SJ, Davis RL, Wright WE, Lassar AB, Weintraub $\mathrm{H}$. Positive autoregulation of the myogenic determination gene MyoD1. Cell 1989;58:241-248.

41. Walters EH, Stickland NC, Loughna PT. The expression of the myogenic regulatory factors in denervated and normal muscles of different phenotypes. J Muscle Res Cell Motil 2000;21:647-653.

42. Sakuma K, Watanabe K, Sano M, Uramoto I, Sakamoto K, Totsuka T. The adaptive response of MyoD family proteins in overloaded, regenerating and denervated rat muscles. Biochim Biophys Acta 1999;1428: 284-292.

43. Zador E, Bottka S, Wuytack F. Antisense inhibition of myoD expression in regenerating rat soleus muscle is followed by an increase in the mRNA levels of myoD, myf-5 and myogenin and by a retarded regeneration. Biochim Biophys Acta 2002;1590:52-63.

44. Kraus B, Pette D. Quantification of MyoD, myogenin, MRF4 and Id-1 by reverse-transcriptase polymerase chain reaction in rat muscles: effects of hypothyroidism and chronic low-frequency stimulation. Eur J Biochem 1997;247:98-106.

45. Sander HW, Saadeh PB, Chandswang N, Greenbaum D, Chovroverty S. Diaphragmatic denervation in intensive care unit patients. Electromyogr Clin Neurophysiol 1999;39:3-5. 